\title{
Finger-Vein Recognition Based on Gabor Features
}

\author{
Jinfeng Yang, Yihua Shi and Renbiao Wu \\ Tianjin Key Lab for Advanced Signal Processing, Civil Aviation University of China \\ China
}

\section{Introduction}

Recently, a new biometric technology based on human finger-vein patterns has attracted the attention of biometrics-based identification research community. Compared with other traditional biometric characteristics (such as face, iris, fingerprint, etc.), finger vein exhibits some excellent advantages in application. For instance, apart from uniqueness, universality, permanence and measurability, finger-vein based personal identification systems hold the following merits:

- Immunity to counterfeit: Finger veins hiding underneath the skin surface make vein pattern duplication impossible in practice.

- Active liveness: Vein information disappears with musculature losing energy, which makes artificial veins unavailable in application.

- User friendliness: Finger-vein images can be captured noninvasively without the contagion and un-pleasant sensations.

Hence, the finger-vein recognition technology is widely considered as the most promising biometric technology in future.

The current available techniques for finger-vein recognition are mainly based on vein texture feature extraction (Miura et al., 2004; 2007; Mulyono and Horng, 2008; Zhang et al., 2006; Vlachos et al., 2008; Yang et al., , 2009a;b;c; Hwan et al., 2009; Liu et al., 2010; Yang et al., 2010). Although texture features are effective for finger-vein recognition, three inherent drawbacks remain unsolved. First, the current finger-vein ROI localization methods are sensitive to finger position variation, which inevitably increases intra-class variation of finger veins. Besides, the current finger-vein image enhancement methods are ineffective to improve the quality of finger-vein images, which is very unhelpful for feature information exploration. Most importantly, the current texture-based finger-vein extraction methods are impotent to reliably describe the properties of veins in orientation and diameter variations, which can directly impair the recognition accuracy.

For finger-vein recognition, a desirable finger-vein feature extraction approach should address ROI localization, image enhancement and oriented-scaled image analysis, respectively. Therefore, in this chapter, detailed descriptions on these aspects are given step by step. First, to localize finger-vein ROIs reliably, a simple but effective ROI segmentation method is proposed based on the physiological structure of a human finger. Second, haze removal method is used to improve the visibility of finger-vein images considering light scattering phenomenon in biological tissues. Third, a bank of even-symmetric Gabor filters is designed to exploit 
finger-vein information in multi-scale and multi-orientation. Finally, to improve the reliability of identification, finger-vein features are extracted in Gabor transform domain, and a fusion scheme in decision level is adopted. Experimental results show that the proposed method performs well in personal identification.

\section{Finger-vein imaging system}

In anatomy, finger veins lie beneath epidermis, and form a network spreading along a finger in a high random manner. Since they are internal, visible lights usually are incapable of imaging them. Thus, illuminating the subcutaneous region of a finger properly is an important task of vein visualization. In medical applications, the NIR (near infrared) lights (760- 850nm) are often used in vein imaging because they can penetrate relatively deep into the skin as well as the radiation of lights can be absorbed greatly by the deoxyhemoglobin (Zharov et al., 2004).

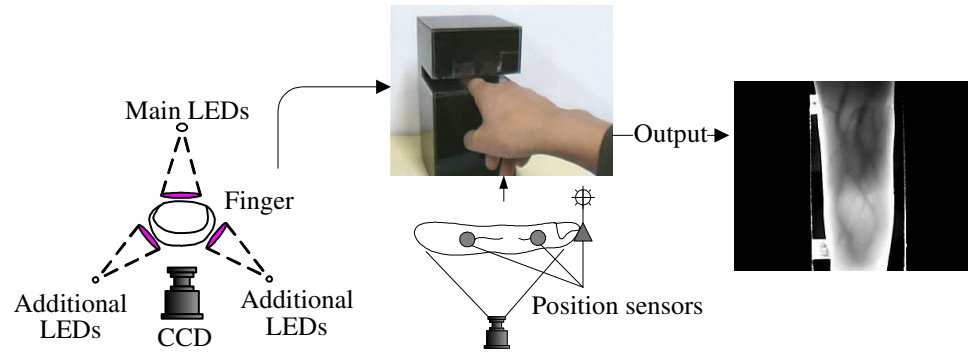

Fig. 1. The proposed principle of a homemade finger-vein imaging system.

In our application, a homemade finger-vein image acquisition system is designed and established as shown in Fig. 1. An open window with a fixed size centered in the width of CCD image plane is set for imaging. The luminaire contains main NIR light-emitting diodes (LEDs) and two additional LEDs at a wavelength of $760 \mathrm{~nm}$, and a CCD sensor is place underneath a finger. Here, the additional LEDs are only used for enhancing the contrast between veins and other tissues. Furthermore, to reduce the variations of imaging poses, two position sensors (denoted by two brighter cylinders in the right of Fig. 1) are set to light an indicator lamp when a finger is placed properly.

From the right of Fig. 1, we can see that the captured image contains not only the finger-vein region but also some uninformative parts. So, the original image needs to be preprocessed to localize a finger-vein region.

\section{Finger-vein image preprocessing}

\subsection{Finger-vein ROI localization}

It is well known that two phalangeal joints, as shown in Fig. 2(a), related with the middle phalanx of a finger make the finger activities possible. And, a functional interphalangeal joint organ is constituted by several components, as shown in Fig. 2(b). Obviously, the density of synovial fluid filling in the clearance between two cartilages is much lower than that of bones. This make possible that more lights penetrate the clearance region when a near infrared LED array is placed over a finger. Thus, a brighter region may exit in the CCD image plane, as shown in Fig. 2(c). Actually, the clearance of a finger inter-phalangeal joint only is with 1.5-2 $\mathrm{mm}$ width. Hence, the brighter region can be substituted by a line with a pixel width. We 


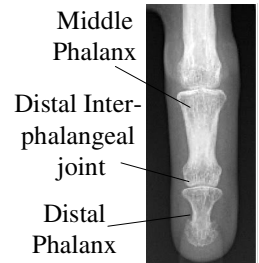

(a)

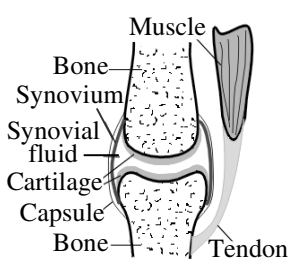

(b)

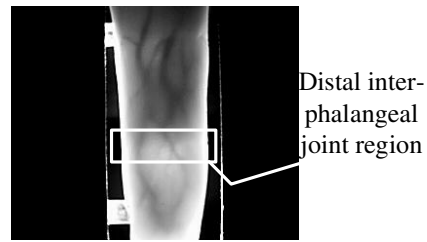

(c)

Fig. 2. Phalangeal joint prior. (a) A X-Ray finger image; (b) Phalangeal joint structure; (c) A possible region (white-rectangle) containing a phalangeal joint.

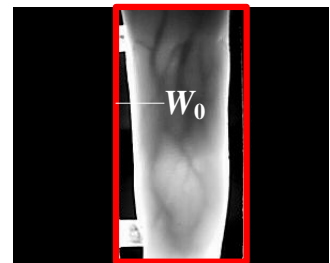

(a)

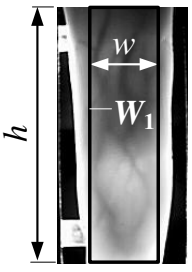

(b)

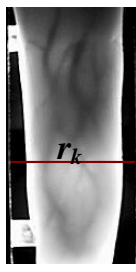

(c)

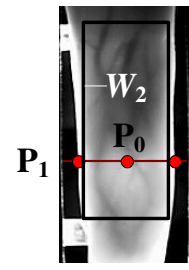

(d)

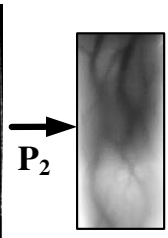

(e)

Fig. 3. Finger-vein ROI localization. (a) Finger-vein imaging window denoted by $W_{0}$; (b) A subwindow $W_{1}$ centered in the width of $W_{0}$; (c) Inter-phalangeal joint position; (d) Finger-vein ROI region $W_{2}$; (e) Finger-vein ROI image.

call the above observation the interphalangeal joint prior. This will be fully used in vein ROI localization.

According to the preceding observation, the idea resides in the use of the distal interphalangeal joint as the localized benchmark. In addition, Yang et al. found out that the only partial imagery of a human finger can deliver discriminating clues for vein recognition (Yang et al., , 2009a). Likewise, we employ the similar subwindow scheme to achieve the description of vein images, since most of vein vessels actually disappear at the finger tip and boundaries. The specific procedure of vein ROI localization is as follows:

- A fixed window (denoted by $W_{0}$ in Fig. 3(a)) same as finger-vein imaging window in size is used to crop a finger-vein candidate region in CCD imaging plane.

- A predefined $w \times h$ window (denoted by $W_{1}$ ) is used to locate a subregion in $W_{0}$. This can reduce the effect of uninformative background, as illustrated in Fig. 3(b);

- The pixel values at each row image are accumulated in the subregion $W_{1}$ :

$$
\Phi_{i}=\sum_{j=1}^{w} I(i, j), \quad i=1, \ldots, h ;
$$

- The maximum row-sum is pinpointed to approximately denote the position ( a line denoted by $r_{k}$ ) of the distal interphalangeal joint, as displayed in Fig. 3(c):

$$
r_{k}=\arg \max _{i \in[1, h]}\left(\Phi_{i}\right)
$$


- Three exemplar points $P_{1}, P_{2}$, and $P_{0}$ are located along the detected baseline. The points $P_{1}$ and $P_{2}$ represent the intersection of the joint baseline and the finger borders, respectively. Meanwhile, the point $P_{0}$ stands for the midpoint of the segment between $P_{1}$ and $P_{2}$;

- Based on point $P_{0}$, a window, denoted by $W_{2}$ in Fig. 3(d), is used to crop a ROI image from the finger vein region as shown in Fig. 3(e). Note that the line $r_{k}$ runs at 2/3 height of $W_{2}$.
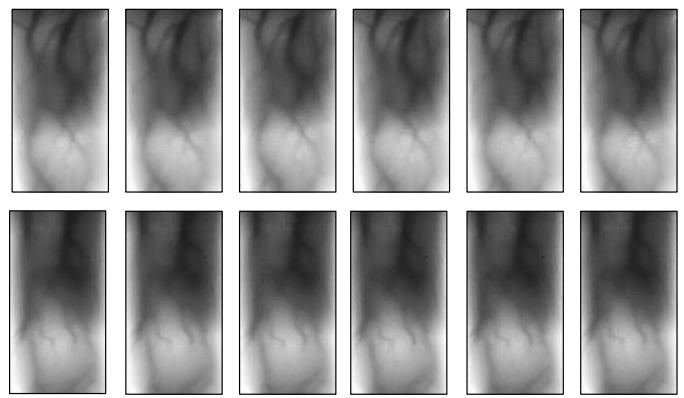

Fig. 4. Some samples ROI images from one subject at different sessions.

The fingers vary greatly in shape not only from different people but also from an identical individual, the cropped ROI by $W_{2}$ therefore may be different in size. For reducing the aspect ratio variation of ROIs, all ROI images are normalized to $180 \times 100$ pixels. Fig. 4 delineates some sample ROI images of one subject at different instants. We can note from Fig. 4 that the sample ROI images have little intra-class variation.

From Fig. 4, we can easily see that the contrast of finger-vein images usually is low and the separability is less between vascular and nonvascular regions. This brings a big challenge for finger-vein recognition, since the finger-vein patterns may be unreliable when feature extraction methods are weak in generalization.

\subsection{Finger-vein image restoration}

Researches in the medical domain reveal that the NIR lights penetrating through a human finger can be absorbed, reflected, scattered and refracted by such finger components as bones, muscles, blood vessels, and skin tissue (Delpy and Cope, 1997; Anderson and Parrish, 1981; Xu et al., 2002). This phenomenon is similar to the way of light scattering in fog (Sassaroli et al., 2004), which can greatly reduce the visibility of imaging scenes. Degraded finger-vein imageries therefore are nature products of the current available finger-vein imaging systems.

To remove the scattering effect from images, dehazing techniques currently are effective ways in many applications (Jean and Nicolas, 2009; Narasimhan and Nayar, 2003). Assume that $I(x, y)$ is the captured image, $R(x, y)$ is the original image free of haze, $\rho(\lambda)$ denotes the extinction coefficient of the fog (scattering medium) and $d(x, y)$ is the depth-map of the scene, the Koschmieder's law (Hautière et al., 2006) defined as the following often is used to restore the degraded image.

$$
R(x, y)=I(x, y) e^{\rho(\lambda) d(x, y)}+I_{v}\left(1-e^{\rho(\lambda) d(x, y)}\right),
$$

where $\lambda$ is wavelength of light and $I_{v}$ is the luminance of the imaging environment. Approximatively, the Koschmieder's law can be transferred to solve the finger-vein image 


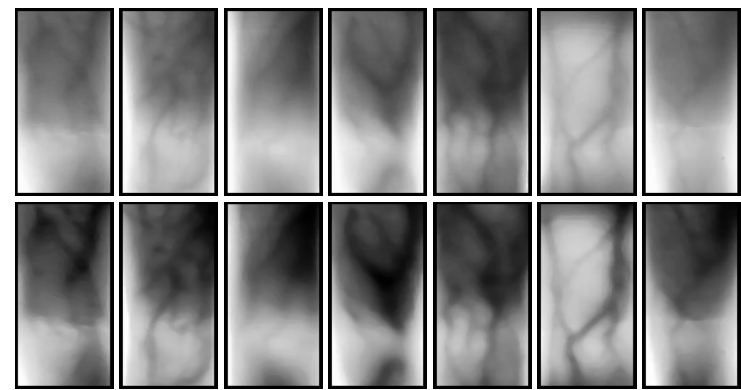

Fig. 5. Dehazing-based image restoration. Top: some original images; Bottom: restored images.

restoration problem since the fog and the biological tissues are two light scattering media with different extinction coefficients corresponding to different light wavelengths.

Inconveniently, it is difficult to obtain the exact $\rho(\lambda), I_{\mathcal{v}}$ and $d(x, y)$ in practice, so a filter approach proposed in (Jean and Nicolas, 2009) is adopted here to estimate $R(x, y)$. This method can successfully implement visibility restoration from a single image with high speed. Fig. 5 shows some low-contrast, degraded finger-vein images and their restored versions. It can be seen from Fig. 5 that haze removal can improve image visibility apparently. However, it is also obvious that the contrast between venous region and nonvenous region is still low, and the brightness is nonuniform in nonvenous region. All these may affect the subsequent processing in feature extraction.

\subsection{Finger-vein image enhancement}

To further improve the contrast of a finger-vein image as well as compensate the nonuniform illumination in an automatic manner, a nonlinear method proposed in (Shi et al., 2007) is first used to correct pixels adaptively, then the illumination variations across the whole image are approximately estimated. From Fig. 5, we can see that venous regions are always darker than nonvenous regions in brightness due to NIR light absorbtion, which is not helpful for making venous region (object region) salient in practice. The negative version of a restored and corrected finger-vein image therefore is used for background illumination estimation, as shown in Fig. 6(b). Here, the average filter with a $16 \times 16$ mask is used as a coarse estimator of the background illumination, as shown in Fig. 6(c).

Subtracting the estimated background illumination from the negative image, we can obtain an image with lighting variation compensation, as shown in Fig. 6(d), Then, we enhance the lighting corrected image by means of histogram equalization. Such processing compensates for the nonuniform illumination, as well as improves the contrast of the image. Fig. 6(e) and 6(f) show the enhanced results of some finger-vein images, from which we can clearly see that the finger-vein network characteristics become clearer than those in the top of Fig. 5. To reduce the noises generated by image operation, the median filter with a $3 \times 3$ mask is used accordingly.

\section{Finger-vein feature analysis}

\subsection{Even Gabor filter design}

Gabor filters have been successfully employed in a wide range of image-analysis applications since they are tunable in scale and orientation (Jie et al., 2007; Ma et al., 2003; Jain et al., 2007; Laadjel et al., 2008; Lee, 1996; Yang et al., 2003; Zhu et al., 2007). Considering the variations 


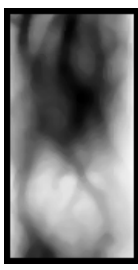

(a)

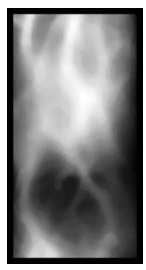

(b)

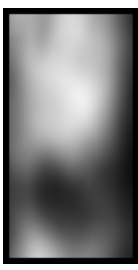

(c)

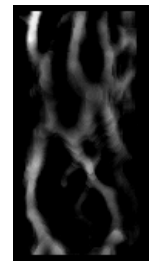

(d)

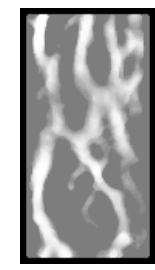

(e)
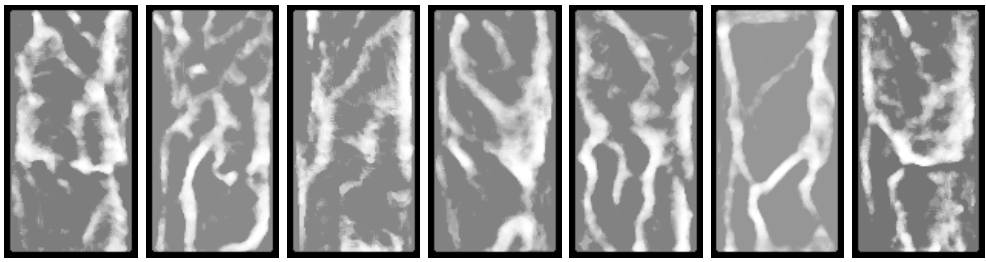

(f)

Fig. 6. Enhancement procedure. (a) Restored finger-vein image; (b) Negative version of the corrected image after restoration; (c) Estimated background illumination; (d) Image with background illumination subtraction; (e) Enhanced image; (f) Other enhanced results corresponding to the samples in Fig. 5.

of vessels in orientation and diameter along a finger, oriented Gabor filters in multiscale are therefore desirable for venous region texture analysis.

A two-dimensional Gabor filter is a function composed by a Gaussian-shaped function and a complex plane wave (Daugman, 1985), which is defined as

$$
G(x, y)=\frac{\gamma}{2 \pi \sigma^{2}} \exp \left\{-\frac{1}{2}\left(\frac{x_{\theta}^{2}+\gamma^{2} y_{\theta}^{2}}{\sigma^{2}}\right)\right\} \exp \left(\hat{j} 2 \pi f_{0} x_{\theta}\right),
$$

where

$$
\left[\begin{array}{l}
x_{\theta} \\
y_{\theta}
\end{array}\right]=\left[\begin{array}{cc}
\cos \theta & \sin \theta \\
-\sin \theta & \cos \theta
\end{array}\right]\left[\begin{array}{l}
x \\
y
\end{array}\right],
$$

$\hat{j}=\sqrt{-1}, \theta$ is the orientation of a Gabor filter, $f_{0}$ denotes the filter center frequency, $\sigma$ and $\gamma$ respectively represent the standard deviation (often called scale) and aspect ratio of the elliptical Gaussian envelope, $x_{\theta}$ and $y_{\theta}$ are rotated versions of the coordinates $x$ and $y$. Determining the values of the four parameters $f_{0}, \sigma \gamma$ and $\theta$ usually play an important role in making Gabor filters suitable for some specific applications (Lee, 1996).

Using Euler formula, Gabor filter can be decomposed into a real part and an imaginary part. The real part, usually called even-symmetric Gabor filter (denoted by $G^{e}(\cdot)$ in this paper), is suitable for ridge detection in an image (Yang et al., 2003), while the imaginary part, usually called odd-symmetric Gabor filter, is beneficial to edge detection (Zhu et al., 2007). Since the finger veins appear dark ridges in image plane, even-symmetric Gabor filter here is used to exploit the underlying features from the finger-vein network. To make even Gabor wavelets into admissible Gabor wavelets, the DC response should be compensated. 
Based on Eq. 4, a bank of admissible even-symmetric Gabor filters subtracting the DC response can be expressed as (Lee, 1996)

$$
G_{s k}^{e}(x, y)=\frac{\gamma}{2 \pi \sigma_{s}^{2}} \exp \left\{-\frac{1}{2}\left(\frac{x_{\theta_{k}}^{2}+\gamma^{2} y_{\theta_{k}}^{2}}{\sigma_{s}^{2}}\right)\right\} \times\left(\cos \left(2 \pi f_{s} x_{\theta_{k}}\right)-\exp \left(-\frac{v^{2}}{2}\right)\right),
$$

where $s$ is the scale index, $k$ is the orientation index and $v$ is a factor determining DC response whose value is determined by $\sqrt{2 \ln 2}\left(2^{\triangle \phi}+1\right) /\left(2^{\triangle \phi}-1\right)$.

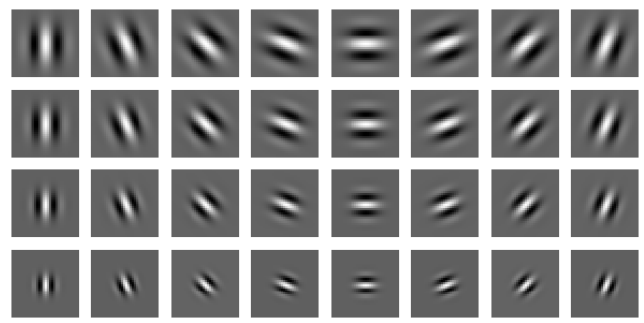

(a)
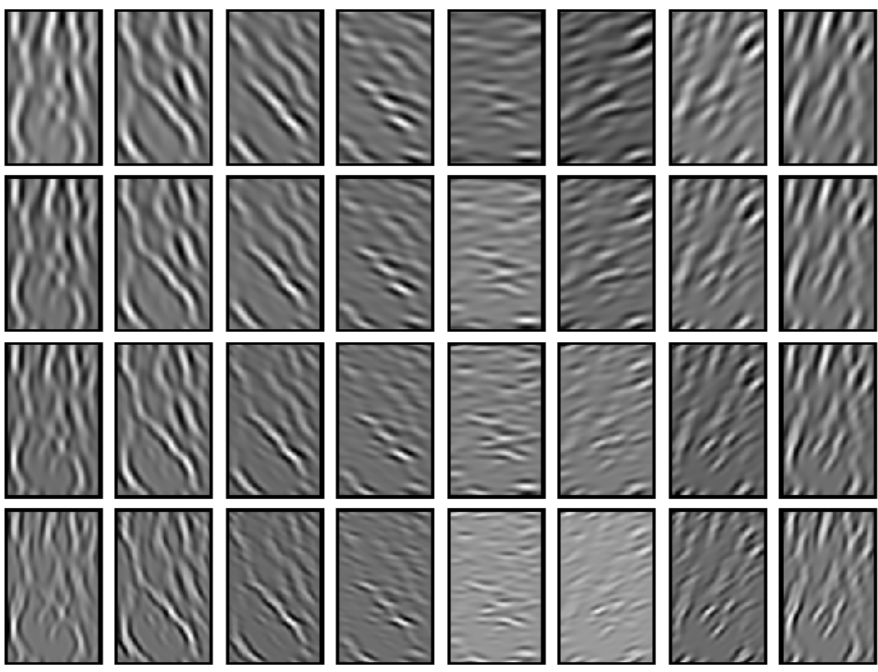

(b)

Fig. 7. Spatial filtering. (a) A bank of even-symmetric Gabor filters; (b) The 2D convolution results.

Since $f_{s}, \sigma_{s}, \gamma$ and $\theta$ usually govern the output of a Gabor filter, these parameters should be determined sensibly for finger-vein analysis application. Considering that vein vessels hold high random characteristics in diameter and orientation, $\gamma$ is set equal to one (i.e., Gaussian function is isotropic) for reducing diameter deformation arising from elliptic Gaussian envelop, $\theta$ varies from zero to $\pi$ with a $\pi / 8$ interval (that is, the even-symmetric Gabor filters are embodied in eight channels). To determine the relation of $\sigma_{s}$ and $f_{s}$, a scheme proposed in 
(Daugman, 1985; Lee, 1996) is used here, which is defined as follow

$$
\sigma_{S} f_{s}=\frac{1}{\pi} \sqrt{\frac{\ln 2}{2}} \cdot \frac{2^{\triangle \phi}+1}{2^{\triangle \phi}-1}
$$

where $\triangle \phi(\in[0.5,2.5])$ denotes the spatial frequency bandwidth (in octaves) of a Gabor filter. Let $s=1, \cdots, 4, \sigma_{s}=8,6,4,2$ and $k=1, \cdots, 8$, we can build a bank of even-symmetric Gabor filters with four scales and eight orientations, as shown in Fig. 7(a). Assume that $F(x, y)$ denote a filtered $R(x, y)$, we can obtain

$$
F_{s k}(x, y)=G_{s k}^{e}(x, y) * R(x, y),
$$

where $*$ denotes 2D image convolution operation. Thus, for a enhanced finger-vein image, 32 filtered images are generated by a bank of Gabor filters, as shown in Fig. 7(b). Noticeably, Gabor filters corresponding to the top row and the bottom row in Fig. 7(a), respectively, are undesirable for finger-vein information exploitation since they can result in losing a lot of vein information due to improper scales. The filtered images with two scales corresponding to the two rows in the middle of Fig. 7(b) therefore are used for finger-vein feature extraction.

\subsection{Finger-vein feature extraction}

According to the above discussion, the outputs of Gabor filters at the sth scale forms an 8 -dimensional vector at each point in $R(x, y)$. For a pixel, its corresponding vector therefore is able to represent its local characteristic. For dimension reduction, an 8-dimensional vector based on the statistical information in a $10 \times 10$ small block of a filtered image is constructed instead of a pixel-based vector. Thus, for a certain scale, $180(18 \times 10)$ vectors can be extracted from the filtered images in Gabor transform domain. Assume that $H_{18 \times 10}$ represent the block matrix of a filter image, the statistics based on a block $H_{i j}$ (a component of $H$ in the $i$ th column and the $j$ th row, where $i=1,2, \cdots, 10$ and $j=1,2, \cdots, 18)$ can be computed. Here, the average absolute deviation from the mean (AAD) (Jain et al., 2000) $\delta_{i j}^{s}$ of the magnitudes of $F_{s k}(x, y)$ corresponding to $H_{i j}$ is calculated as

$$
\left\{\begin{array}{l}
\delta_{i j}^{s k}=\frac{1}{R} \sum_{H_{i j}}|| F_{s k}(x, y)\left|-\mu_{i j}^{s k}\right| \\
\mu_{i j}^{s k}=\frac{1}{R} \sum_{H_{i j}}\left|F_{s k}(x, y)\right|
\end{array}\right.
$$

where $K$ is the number of pixels in $H_{i j}, \mu_{i j}^{s k}$ is the mean of the magnitudes of $F_{s k}(x, y)$ in $H_{i j}$. Based on Eq. 8, the local statistics of filtered images are shown in Fig. 8, where the statistical information in a red box is used for finger-vein feature analysis.

Thus, the vector matrix at the sth scale of Gabor filter can be represented by

$$
\mathbf{V}_{s}=\left[\begin{array}{ccc}
\vec{v}_{11}^{s} & \cdots & \vec{v}_{1 N}^{s} \\
\vdots & \vec{v}_{i j}^{s} & \vdots \\
\vec{v}_{M 1}^{s} & \cdots & \vec{v}_{M N}^{s}
\end{array}\right]_{18 \times 10}
$$

where $\vec{v}_{i j}^{s}=\left[\delta_{i j}^{s 1}, \cdots, \delta_{i j}^{s k}, \cdots, \delta_{i j}^{s 8}\right]$. According to Eq. 9, uniting all the vectors together can form a $2880(180 \times 2 \times 8)$ dimensional vector, which is not beneficial for feature matching. 


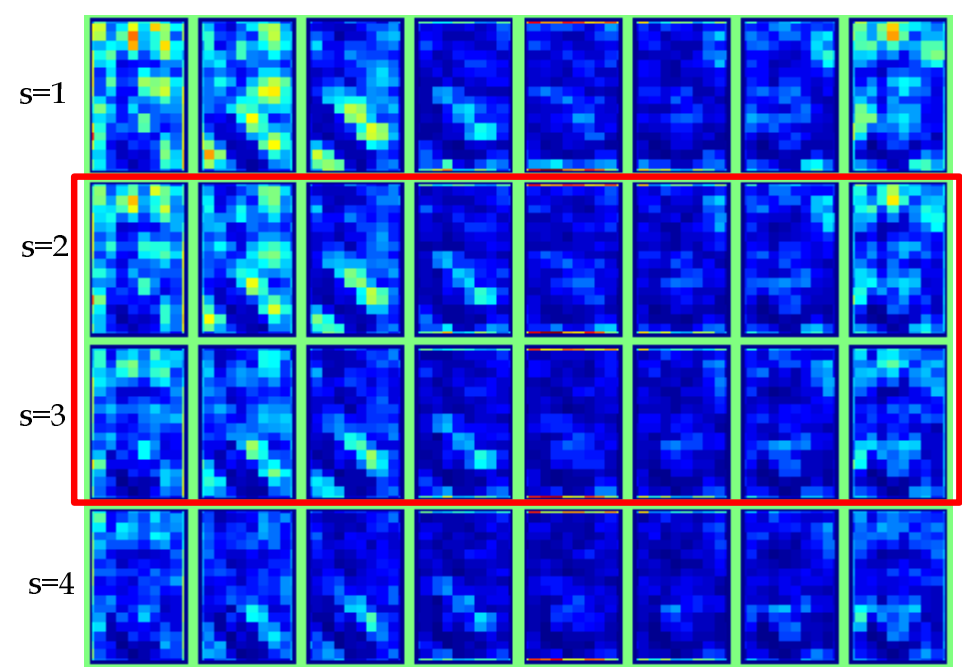

Fig. 8 . The average absolute deviations (AADs) in $[18 \times 10] \times 8$ blocks of the filtered finger-vein images in different scales and orientations.

Hence, based on $\mathbf{V}_{s}$, two new feature matrixes are constructed as

$$
\mathbf{U}_{s}=\left[\begin{array}{ccccc}
\left\|\vec{v}_{11}^{s}\right\| & \cdots & \left\|\vec{v}_{1 j}^{s}\right\| & \cdots & \left\|\vec{v}_{1 N}^{s}\right\| \\
\vdots & \vdots & \vdots & \vdots & \vdots \\
\left\|\vec{v}_{i 1}^{s}\right\| & \cdots & \left\|\vec{v}_{i j}^{s}\right\| & \cdots & \left\|\vec{v}_{i N}^{s}\right\| \\
\vdots & \vdots & \vdots & \vdots & \vdots \\
\left\|\vec{v}_{M 1}^{s}\right\| & \cdots & \left\|\vec{v}_{M j}^{s}\right\| & \cdots & \left\|\vec{v}_{M N}^{s}\right\|
\end{array}\right],
$$

and

$$
\mathbf{Q}_{s}=\left[\begin{array}{ccccc}
\alpha_{1(1,2)}^{S} & \cdots & \alpha_{1(j, j+1)}^{S} & \cdots & \alpha_{1(N-1, N)}^{S} \\
\vdots & \vdots & \vdots & \vdots & \vdots \\
\alpha_{i(1,2)}^{\mathcal{S}} & \cdots & \alpha_{i(j, j+1)}^{s} & \cdots & \alpha_{i(N-1, N)}^{\mathcal{S}} \\
\vdots & \vdots & \vdots & \vdots & \vdots \\
\alpha_{M(1,2)}^{S} & \cdots & \alpha_{M(j, j+1)}^{S} & \cdots & \alpha_{M(N-1, N)}^{S}
\end{array}\right],
$$

where $M=18, N=10,\|\bullet\|$ denotes the Euclidean norm of a vector, $\alpha_{i(j, j+1)}^{s}$ is the angle of two adjacent vectors in the $i$ th row. In this way, matrix $\mathbf{U}_{s}$ is suitable for local feature representation, and matrix $\mathbf{Q}_{s}$ is suitable for global feature representation. Hence, using $\mathbf{U}_{s}$ and $\mathbf{Q}_{s}$, the local and global characteristics of a finger-vein image in the Gabor transform domain at the sth scale can be described sensibly and reliably. For convenience, the components of matrix $\mathbf{U}_{s}$ and $\mathbf{Q}_{s}$ are respectively rearranged by rows to form two $1 D$ 
feature vectors, here called FVCodes,

$$
\begin{aligned}
& Z_{s}^{U}=\left[\left\|\vec{v}_{11}^{s}\right\|, \cdots,\left\|\vec{v}_{i j}^{s}\right\|, \cdots,\left\|\vec{v}_{M N}^{s}\right\|\right]^{T} \\
& Z_{s}^{Q}=\left[\alpha_{1(1,2)}^{s}, \cdots, \alpha_{i(j, j+1,}^{s}, \cdots, \alpha_{M(N-1, N)}^{s}\right]^{T} . \\
& (i=1,2, \cdots, M ; j=1,2, \cdots, N ; s=2,3)
\end{aligned}
$$

Since the components in $\mathbf{U}_{s}$ and $\mathbf{Q}_{s}$ are not of the same order of magnitude, it is not advisable to combine $Z_{S}^{U}$ and $Z_{S}^{Q}$ together for feature simplification in practices.

\section{Finger-vein recognition}

\subsection{Finger-vein classification}

As face, iris, and fingerprints recognition, finger-vein recognition is also based on pattern classification. Hence, the discriminability of the proposed FVCodes determines their reliability in personal identification. To test the discriminability of the extracted FVCodes at a certain scale, the cosine similarity measure classifier (CSMC) is adopted here for classification. The classifier is defined as

$$
\left\{\begin{array}{l}
\tau=\arg \min _{Z_{s}^{*} \in C_{\kappa}} \varphi\left(Z_{s}, Z_{s}^{\cdot k}\right) \\
\varphi\left(Z_{S}, Z_{S}^{\cdot \mathcal{k}}\right)=1-\frac{Z_{s}^{\cdot T} Z_{s}^{\cdot k}}{\left\|Z_{s}^{\cdot}\right\|\left\|Z_{s}^{\cdot k}\right\|}
\end{array},\right.
$$

where $Z_{S}$ and $Z_{S}^{\cdot \mathcal{K}}$ respectively denote the feature vector of an unknown sample and the $\kappa$ th class, $C_{\kappa}$ is the total number of templates in the $\kappa$ th class, $\|\bullet\|$ indicates the Euclidean norm, and $\varphi\left(Z_{S^{\prime}}, Z_{S}^{\cdot \mathcal{K}}\right)$ is the cosine similarity measure. Using similarity measure $\varphi\left(Z_{S_{S}}, Z_{S}^{\cdot \mathcal{K}}\right)$, the feature vector $Z_{s}$ is classified into the $\tau$ th class.

\subsection{Decision-level fusion}

According to section 4, we can see that the proposed FVCodes are different in content, dimension and scale for finger-vein feature description. Therefore, fusion of the matching results based on $Z_{s}$ may improve the performance of identification. Nowadays, many approaches have been proposed in multi-biometrics fusion, such as Bayes algorithm, KNN classifier, OS-Rule, SVM classifier, decision templates algorithm, Dempster-Shafer (D-S) algorithm. Compared to other approaches, the D-S evidence theory works better in integrating multiple evidences for decision making. Details on D-S theory can be found in (Ren et al., 2009; Yager, 1987; Brunelli et al., 1995). Here, a overview of D-S theory is given briefly in the following.

Let $\Theta=\left\{\theta_{1}, \cdots, \theta_{n}\right\}$ be a frame of discernment, the power set $2^{\Theta}$ be the set of $2^{n}$ propositions (subsets) of $\Theta$. For an individual proposition $A$ (or an evidence), $m(A)$ is defined as basic belief assignment function (or mass function) if

$$
\left\{\begin{array}{l}
\sum_{A \in \Theta} m(A)=1 \\
m(\varnothing)=0
\end{array} .\right.
$$

For a subset $A$ satisfying $m(A)>0$ is called focal element. Now, given two evidence sets $E_{1}$ and $E_{2}$ from $\Theta$ with belief functions, $m_{1}(\cdot)$ and $m_{2}(\cdot)$, let $A_{i}$ and $B_{i}$ be two focal elements respectively corresponding to $E_{1}$ and $E_{2}$, the combination of the two evidences is given by 
Dempster's orthogonal operator

$$
m(A)=\left\{\begin{array}{ll}
\frac{\sum_{A_{i} \cap B_{j}=A} m_{1}\left(A_{i}\right) m_{2}\left(B_{j}\right)}{1-K_{a}}, & A \neq \varnothing, \\
0, & A=\varnothing
\end{array},\right.
$$

where

$$
K_{a}=\sum_{A_{i} \cap B_{j}=\varnothing} m_{1}\left(A_{i}\right) m_{2}\left(B_{j}\right)
$$

represents the conflict degree between two evidence sets. Traditionally, $K_{a}$ usually leads to unimagined decision-making if it increases to a certain limit. Aiming to weaken the degree of evidence confliction, we have proposed an improved scheme in (Ren et al., 2009), and obtained better fusion results for fingerprint recognition. In view of finger-vein recognition application, a scheme based on D-S theory, as shown in Fig. 9, here is adopted to implement fusion in decision level.

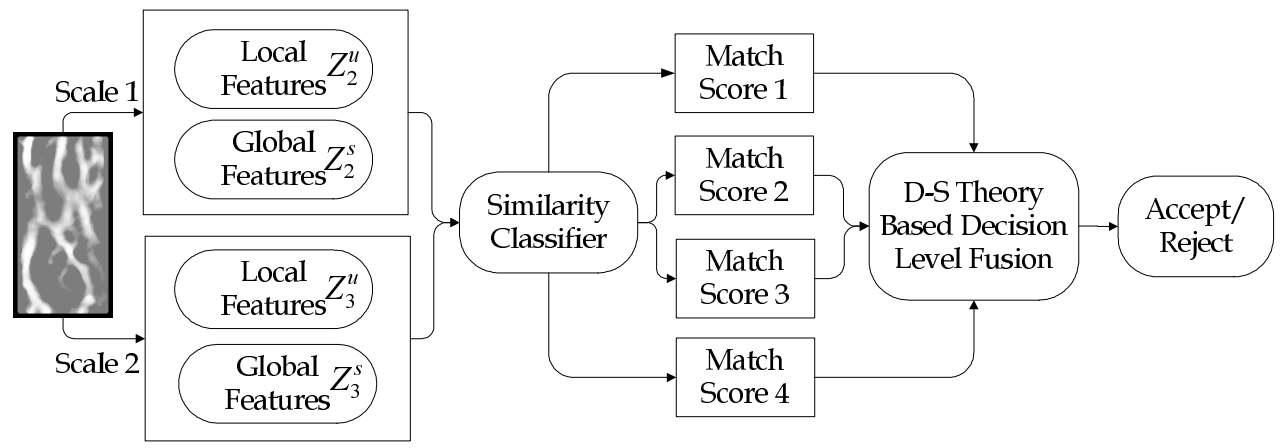

Fig. 9. The scheme of decision level fusion based on D-S theory.

First, for a certain extracted vector $Z_{s}$, match scores can be generated using CSMC. Based on the match scores, a basic belief assignment construction method proposed in (Ren et al., 2009) is then used for mass function formation. Thus, for a proposition $A$, mass function of each evidence is combined by

$$
m(A)=\left(m_{1} \oplus m_{2} \oplus m_{3} \oplus m_{4}\right)(A)
$$

where $\oplus$ represents the improved D-S combination rule proposed in (Ren et al., 2009), and $m_{1}$, $m_{2}, m_{3}$ and $m_{4}$ are the mass functions respectively computed from different evidence-match results using CSMC. The belief and plausibility committed to $A, \operatorname{Bel}(A)$ and $\operatorname{Pl}(A)$, can be computed as

$$
\left\{\begin{array}{l}
\operatorname{Bel}(A)=\sum_{B \subset A} m(B) \\
\operatorname{Pl}(A)=\sum_{B \cap A \neq \varnothing} m(B)
\end{array},\right.
$$

where $\operatorname{Bel}(A)$ represents the lower limit of probability and $\mathrm{Pl}(A)$ represents the upper limit. To give a reasonable decision, accept/reject, for incoming samples, an optimal threshold value related to the evidence mass functions should be found during training phase. 


\section{Experiments}

\subsection{Finger-vein image database}

Because of the vacancy of common finger-vein image database for finger-vein recognition, we build an image database which contains 4500 finger-vein images from 100 individuals. Each individual contributes 45 finger-vein images from three different fingers: forefinger, middle finger and ring finger (15 images per finger) of the right hand. All images are captured using a homemade image acquisition system, as shown in Fig. 1. The captured finger-vein images are 8-bit gray images with a resolution of $320 \times 240$.

\subsection{Performance evaluation of FVCode}

Due to the high randomicity of the finger-vein networks, the discriminability of the proposed FVCodes may embody not only in different individuals but also in different fingers of an identical individual. So, to investigate the differences among forefinger, middle finger and ring finger, 5 finger-vein images from one finger are selected as testing samples while the rest as training. Since the dimension of a FVCode is not high $(\leq 180)$, dimension reduction is not necessary for improving match efficiency. Moreover, the integrality of FVCodes describing finger-vein networks may be destroyed by dimension reduction. Therefore, the extracted FVCodes are directly used by CSMC for finger classification, some classification results are listed in Tables 1 and 2, where F_finger, M_finger and R_finger, respectively represent forefinger, middle finger and ring finger, and FRR and FAR respectively represent false rejection rate and false acceptance rate.

\begin{tabular}{|c|c|c|c|c|c|}
\hline L-FVCodes & & F-finger(500 & M-finger(500 & R-finger(500) & FAR(\%) \\
\hline \multirow{3}{*}{$s=2$} & \begin{tabular}{|l|} 
F-finger \\
\end{tabular} & $492(98.4 \%)$ & 5 & 7 & 1.2 \\
\hline & M-finger & 6 & $489(97.8 \%)$ & 10 & 1.6 \\
\hline & R-finger & 2 & 6 & $483(96.6 \%)$ & 0.8 \\
\hline \multirow{3}{*}{$s=3$} & F-finger & $490(98.0 \%)$ & 8 & 5 & 1.3 \\
\hline & M-finger & 6 & $486(97.2 \%)$ & 13 & 1.9 \\
\hline & R-finger & 4 & 6 & $482(96.4 \%)$ & 1.0 \\
\hline FRR & $\mathrm{s}=2$ & 1.6 & 2.2 & 3.4 & \\
\hline$(\%)$ & $s=3$ & 2.0 & 2.8 & 3.6 & \\
\hline
\end{tabular}

Table 1. Finger-vein image classification results using local FVCodes.

\begin{tabular}{|c|c|c|c|c|c|}
\hline G-FVCodes & & F-finger(500) & $\bar{M}$-finger(50c & R-finger(500) & FAR(\%) \\
\hline \multirow{3}{*}{$s=2$} & F-finger & $488(97.6 \%)$ & 10 & 8 & 1.8 \\
\hline & M-finger & 8 & $483(96.6 \%)$ & 13 & 2.1 \\
\hline & $\mathrm{R}$-finger & 4 & 7 & $479(95.8 \%)$ & 1.1 \\
\hline \multirow{3}{*}{$s=3$} & F-finger & 492(98.4\%) & 4 & 8 & 1.2 \\
\hline & M-finger & 5 & $489(97.8 \%)$ & 9 & 1.4 \\
\hline & $\mathrm{R}$-finger & 3 & 7 & $483(96.6 \%)$ & 1.0 \\
\hline FRR & $\mathrm{s}=2$ & 2.4 & 3.4 & 4.2 & \\
\hline$(\%)$ & $\mathrm{s}=3$ & 1.6 & 2.2 & 3.4 & \\
\hline
\end{tabular}

Table 2. Finger-vein image classification results using global FVCodes.

From Tables 1 and 2, we can clearly see that forefingers hold the best capability in classification, while middle fingers appear better than ring fingers in correct classification rate (CCR) but lower than ring fingers in FAR. Moreover, for all test samples, local FVCodes can 
achieve CCRs of $97.6 \%$ and $97.2 \%$ at two scales, which shows that the discriminability of local FVCodes decreases with scale $\left(\sigma_{2}>\sigma_{3}\right)$. On the contrary, the discriminability of global FVCodes increases with scale since CCR increases from $96.67 \%$ at second scale $\left(\sigma_{2}\right)$ to $97.6 \%$ at third scale $\left(\sigma_{3}\right)$. These results show that 1 ) not only the proposed FVCode exhibits significant discriminability but also every finger is suitable for personal identification, and 2) fusion of local and global FVCodes in this two scales can improve the performance of finger-vein features in personal identification. Hence, the finger-vein images from different fingers can be viewed as from different individuals, and the proposed fusion scheme, as shown in Fig. 9, is desirable for recognition performance improvement. In the subsequent experiments, the database thus is expanded manually to 600 subjects and 15 finger-vein images per subject.

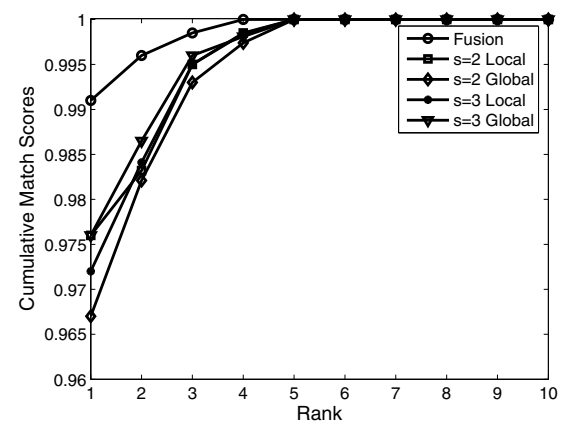

(a) Identification

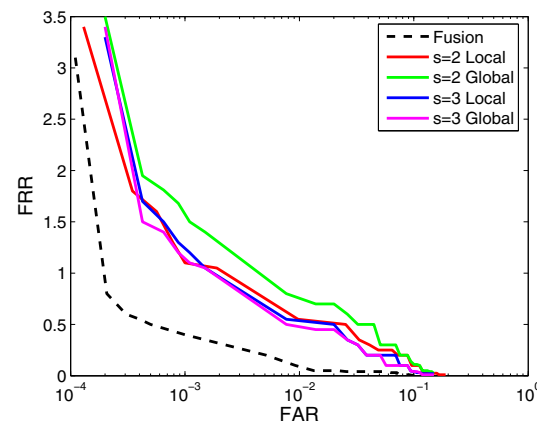

(b) Verification

Fig. 10. The results of identification and verification

To obtain an unbiased estimate of the true recognition rate, a leave-one-out cross-validation method is used here. That is, leaving one example out sequentially and training on the rest accordingly, we conduct a classification of the omitted example. Consider that cumulative match scores (CMS) proposed in (Phillips et al., 2000) be more general in measuring classification performance, we therefore use it to evaluate the proposed finger-vein recognition algorithm. CMS can report the correct match probability (CMP) corresponding to the ranked $n$ matches, and CCR is equivalent to the first CMP (rank =1). Fig. 10 demonstrates the performance of the proposed method in identification and verification (for ranks up to 10). From Fig. 10, we can see that both local FVCodes and global FVCodes have their own merits in finger-vein recognition. Results from local FVCodes at the first scale $(s=2)$ are somewhat better than those at the second $(s=3)$, whereas this situation is reverse in results from global FVCodes. This is because, in the Gabor transform domain, feature variations are sufficiently represented locally at a big scale and globally at a small scale. Furthermore, the performance of both identification and verification for decision-level fusion is improved better, especially in FAR. This demonstrates that fusion of FVCodes at the two scales can improve the reliability of identification significantly. Hence, the finger-vein recognition technology is worthwhile to pay further attentions in security.

\subsection{Comparison with existing methods}

Unlike iris, face and fingerprints, research on finger-vein recognition is in an initial stage. For the purpose of comparison, we only implement some methods according to published papers (Miura et al., 2004; 2007; Mulyono and Horng, 2008; Zhang et al., 2006). Since it is difficult to obtain the detailed descriptions of the existing techniques in finger-vein recognition, only 
algorithms on finger-vein feature extraction are implemented faithfully to the originals. The cosine similarity classifier here is used as a common measure to test the performance of the extracted finger-vein features. This is helpful to illustrate the capabilities of the existing finger-vein features in discriminability.

However, since some conditions may be uncertain in practice, our implemented versions may be inferior to the originals. Therefore, the comparison results only show the performance of previous methods approximately. Based on the expanded database (600 subjects, 15 images per subject), using 10 image of each subject as training samples and the rest as testing samples, we give the CCRs and FARs in Table 3.

\begin{tabular}{|c|ccccc|}
\hline$(\%)$ & Miura('04) & Zhang('06) & Miura('07) & Lian('08) & L-FVCode $(s=2)$ \\
\hline CCR & 90.63 & 90.97 & 93.37 & 89.43 & 97.6 \\
FAR & 9.68 & 9.27 & 7.21 & 12.21 & 1.2 \\
\hline
\end{tabular}

Table 3. Comparison results of the existing methods.

From Table 3, we can see that the proposed method is better than the previous in CCR and FAR. This is exciting indeed. Unfortunately, the results from the previous methods are significantly lower than those reported in the originals, especially in FARs. We think that two main reasons are responsible for this situation. First, the used image databases are different, which can directly lead to experimental deviations in practice. Second, the qualities of the used images may be different, that is to say, different image sensors generated different image qualities, which can significantly degrade different algorithms in finger-vein extraction. Hence, to make finger-vein recognition technology progress steadily, a standard finger-vein image database is indispensable in finger-vein based research community.

\section{Conclusion}

A method of personal identification based on finger-vein recognition has been discussed elaborately in this chapter. First, a stable finger-vein ROI localization method was introduced based on an interphalangeal joint prior. This is very important for finger-vein based practical application. Second, haze removal was adopted to restore the degraded finger-vein images, and background illumination was compensated from illumination estimation. Third, a bank of Gabor filters were designed to exploit the underlying finger-vein characteristics, and both local and global finger-vein features were extracted to form FVCodes. Finally, finger-vein classification was implemented using the cosine similarity classifier, and a fusion scheme in decision level was adopted to improve the reliability of identification. Experimental results have shown that the proposed method performed well in personal identification.

Undoubtedly, the method discussed in this chapter can not be optimal in accuracy and efficiency considering the development of finger-vein recognition technology. Therefore, this work should be just for reference to implement a finger-vein recognition task.

\section{Acknowledgements}

This work is jointly supported by NSFC (Grant No.61073143) and TJNSF (Grant No. 07ZCKFGX03700).

\section{References}

Zharov, V.; Ferguson,S.; Eidt, J.; Howard, P.; Fink, L.; \& Waner, M. (2004). Infrared Imaging of Subcutaneous Veins.Lasers in Surgery and Medicine, Vol.34, No.1, (January 2004), pp.56-61, ISSN 1096-9101 
Miura, N. \& Nagasaka, A. (2004). Feature Extraction of Finger-Vein Pattern Based on Repeated Line Tracking and Its Application to Personal Identification. Sachine Vision and Applications, Vol.15, No.4, (October 2004), pp.194-203, ISSN 0932-8092

Miura, N., Nagasaka, A. \& Miyatake, T. (2007). Extraction of Finger-Vein Patterns Using Maximum Curvature Points in Image Profiles. IEICE - Transaction on Information and Systems, Vol.E90-D, No.8, (August 2007), pp.1185-1194, ISSN 0916-8532

Mulyono, D. \& Horng S.J. (2008). A Study of Finger Vein Biometric for Personal Identification. Proceedings of International Symposium on Biometrics and Security Technologies, pp.1-8, ISBN 978-1-4244-2427-6, Islamabad, Pakistan, April 23-24, 2008

Zhang, Z.; Ma, S. \& Han, X. (2006). Multiscale Feature Extraction of Finger-Vein Patterns Based on Curvelets and Local Interconnection Structure Neural Network. Proceedings of 18th International Conference on Pattern Recognition, pp.145-148, ISBN 0-7695-2521-0, Hongkong, China, August 20-24, 2006

Vlachos, M. \& Dermatas, E. (2008). Vein Segmentation in Infrared Images Using Compound Enhancing and Crisp Clustering. Lecture Notes in Computer Science, Vol.5008,(May 2008), pp.393-402, ISSN 0302-9743

Jie, Z.; Ji, Q. \& Nagy, G. (2007). A Comparative Study of Local Matching Approach for Face Recognition. IEEE Transaction on Image Processing, Vol.16, No.10, (October 2007), pp.2617-2628, ISSN 1057-7149

Ma, L.; Tan, T.; Wang, Y. \& Zhang, D. (2003). Personal Identification Based on Iris Texture Analysis. IEEE Transaction on Pattern Analysis and Machine Intelligence, Vol.25, No.12, (December 2003), pp.1519-1533, ISSN 0162-8828

Jain, A.K.; Chen, Y. \& Demirkus, M. (2007). Pores and Ridges: High-Resolution Fingerprint Matching Using Level 3 Features. IEEE Transaction on Pattern Analysis and Machine Intelligence, Vol.29, No.1, (Janary 2007) pp.15-27, ISSN 0162-8828

Laadjel, M.; Bouridane, A.; Kurugollu, F. \& Boussakta, S. (2008). Palmprint Recognition Using Fisher-Gabor Reature Extraction. Proceedngs of IEEE International Conference on Acoustics, Speech and Signal Processing, pp.1709-1712, ISSN 1520-6149, Las Vegas, USA, March 31-April 4, 2008

Shi, Y.H.; Yang, J.F. \& Wu, R.B. (2007). Reducing Illumination Based on Nonlinear Gamma Correction. Proceedings of IEEE International Conference on Image Processing,pp.529-532, ISBN 978-1-4244-1437-6, San Antonio, Texas, USA, September 16-19, 2007

Yang, J.F.; Shi, Y.H. \& Yang, J.L. (2009). Finger-vein Recognition Based on a Bank of Gabor Filters. Proceedings of Acian Conference on Computer Vision, pp.374-383, ISBN 978-3-642-12306-1, Xi'an, China, September 23-27, 2009

Yang, J.F.; Yang, J.L. \& Shi, Y.H. (2009). Finger-vein segmentation based on multi-channel even-symmetric gabor filters. Proceedings of IEEE International Conference on Intelligent Computing and Intelligent Systems, pp.500-503, ISBN 978-1-4244-4754-1 Shanghai, China, November 20-22, 2009

Yang, J.F.; Yang, J.L. \& Shi, Y.H. (2009). Combination of gabor wavelets and circular gabor filter for finger-vein extraction. Lecture Notes in Computer Science, Vol.5754, (September 2009), pp.346-354, ISSN 0302-9743

Choi, J.H.; Song, W.; Kim, T.; Lee, S. \& Kim, H.C. (2009). Finger veinextraction using gradient normalization and principal curvature. Proceedings of the SPIE, Vol.7251, pp.1-9,ISBN 978-0-8194-7501-5, San Jose, USA, January 21, 2009

Liu, Z.; Yin, Y.; Wang, H.; Song, S. \& Li, Q. (2010). Finger Vein Recognition with Minifold Learning. Jounal of Network and Computer Application, Vol.33, No.3, (May 2010), pp.275-282, ISSN 1084-8045 
Yang, J.F. \& Li, X. (2010). Efficient Finger Vein Localization and Recognition. Proceedings of 20th International Conference on Pattern Recognition, pp.1148-1151, ISBN: 978-1-4244-7542-1, Istanbul, Turkey, Aug. 23-26, 2010.

Ren, X.H.; Yang, J.F.; Li, H.H. \& Wu, R.B. (2009). Multi-fingerprint Information Fusion for Personal Identification Based on Improved Dempster-Shafer Evidence Theory. Proceedings of IEEE International Conference on Electronic Computer Technology, pp.281-285, ISBN 978-0-7695-3559-3, Macau, China, February 20-22, 2009

Yager, R. (1987). On the Dempster-Shafer Framework and New Combination Rules, Information Sciences, Vol.41, No.2, (March 1987), pp.93-138, ISSN 0020-0255

Brunelli, R. \& Falavigna, D. (1995). Person Identification Using Multiple Cues. IEEE Transaction on Pattern Analysis and Machine Intelligence, Vol.17, No.10, (October 1995), pp.955-966, ISSN 0162-8828

Daugman, J.G. (1985). Uncertainty Relation for Resolution in Space, Spatial Frequency, and Orientation Optimized by 2D Visual Cortical Filters. Journal of the Optical Society of America, Vol.2, No.7, (July 1985), pp.1160-1169, ISSN 1520-8532

Lee, T.S. (1996). Image Representation Using 2D Gabor Wavelets, IEEE Transaction on Pattern Analysis and Machine Intelligence, Vol.18, No.10, (October 1996), pp.1-13, ISSN 0162-8828

Yang, J.; Liu, L.; Jiang, T. \& Fan, Y. (2003). A Modified Gabor Filter Design Method for Fingerprint Image Enhancement. Pattern Recognition Letters, Vol.24, No.12, (August 2003), pp.1805-1817, ISSN 0167-8655

Zhu, Z.; Lu, H. \& Zhao, Y. (2007). Scale Multiplication in Odd Gabor Transform Domain for Edge Detection. Journal of Visual Communication and Image Representation, Vo.18, No.1, ( February 2007), pp.68-80, ISSN 1047-3203

Jain, A. K.; Prabhakar, S.; Hong, L.; Pankanti, S. (2000). Filterbank-Based Fingerprint Matching. IEEE Transaction on Image Processing, Vol.9, No.5, (May 2000), pp.846-859, ISSN 1057-7149

Phillips, J.; Moon, H.; Rizvi, S. \& Rause, P. (2000). The FERET Evaluation Methodology for Face Recognition Algorithms. IEEE Transaction on Pattern Analysis and Machine Intelligence, Vol.22, No.10, (October 2000), pp.1090-1104, ISSN 0162-8828

Delpy, D.T. \& Cope, M. (1997). Quantification in Tissue Near-Infrared Spectroscopy. Philosophical Transactions of the Royal Society of London, B, Vol.352, No.1354, (June 1997), pp.649-659, ISSN 1471-2970

Anderson, R. \& Parrish, J. (1981). The Optics of Human Skin. Journal of Investigative Dermatology , Vol.77, no.1, (July 1981), pp.13-19, ISSN 0022-202X

Xu, J.; Wei, H.; Li, X.; Wu, G. \& Li, D. (2002). Optical Characteristics of Human Veins Tissue in Kubelka-Munk Model at He-Ne Laser in Vitro. Journal of OptoelectronicsLaser, Vol.13, No.3, (March 2002), pp.401-404, ISSN 1005-0086

Sassaroli, A. et al. Near-infrared spectroscopy for the study of biological tissue. Available from http://ase.tufts.edu/biomedical/research/fantini/researchAreas /NearInfraredSpectroscopy.pdf

Hautière, N.; Tarel, J.P.; Lavenant, J. \& Aubert, D. (2006). Automatic Fog Detection and Estimation of Visibility Distance through Use of an Onboard Camera. Machine Vision and Applications, Vol.17, No.1, (January 2006), pp.8-20, ISSN 0932-8092

Jean-Philippe, T. \& Nicolas, H. (2009). Fast Visibility Restoration from a Single Color or Gray Level Image. Proceedings of International Conference on Computer Vision, pp.2201-2208, ISBN 978-1-4244-4420-5, Kyoto, Japan, Septmber 29-October 2, 2009

Narasimhan, S.G. \& Nayar, S.K. (2003). Contrast Restoration of Weather Degraded Images. IEEE Transaction on Pattern Analysis and Machine Intelligence, Vol.25, No.6, (June 2003), pp.713-724, ISSN 0162-8828 


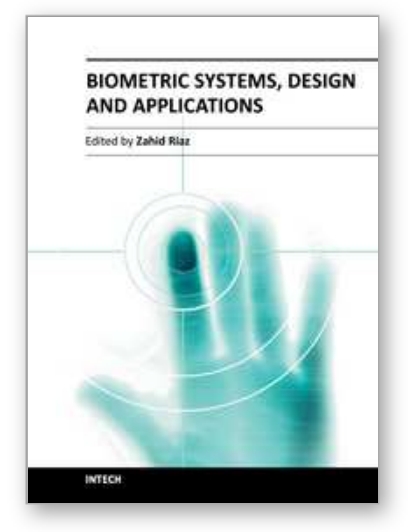

\author{
Biometric Systems, Design and Applications \\ Edited by Mr Zahid Riaz
}

ISBN 978-953-307-542-6

Hard cover, 262 pages

Publisher InTech

Published online 21, October, 2011

Published in print edition October, 2011

Biometric authentication has been widely used for access control and security systems over the past few years. The purpose of this book is to provide the readers with life cycle of different biometric authentication systems from their design and development to qualification and final application. The major systems discussed in this book include fingerprint identification, face recognition, iris segmentation and classification, signature verification and other miscellaneous systems which describe management policies of biometrics, reliability measures, pressure based typing and signature verification, bio-chemical systems and behavioral characteristics. In summary, this book provides the students and the researchers with different approaches to develop biometric authentication systems and at the same time includes state-of-the-art approaches in their design and development. The approaches have been thoroughly tested on standard databases and in real world applications.

\title{
How to reference
}

In order to correctly reference this scholarly work, feel free to copy and paste the following:

Jinfeng Yang, Yihua Shi and Renbiao Wu (2011). Finger-Vein Recognition Based on Gabor Features, Biometric Systems, Design and Applications, Mr Zahid Riaz (Ed.), ISBN: 978-953-307-542-6, InTech, Available from: http://www.intechopen.com/books/biometric-systems-design-and-applications/finger-vein-recognitionbased-on-gabor-features

\section{INTECH}

open science | open minds

\author{
InTech Europe \\ University Campus STeP Ri \\ Slavka Krautzeka 83/A \\ 51000 Rijeka, Croatia \\ Phone: +385 (51) 770447 \\ Fax: +385 (51) 686166 \\ www.intechopen.com
}

\author{
InTech China \\ Unit 405, Office Block, Hotel Equatorial Shanghai \\ No.65, Yan An Road (West), Shanghai, 200040, China \\ 中国上海市延安西路65号上海国际贵都大饭店办公楼 405 单元 \\ Phone: +86-21-62489820 \\ Fax: $+86-21-62489821$
}


(C) 2011 The Author(s). Licensee IntechOpen. This is an open access article distributed under the terms of the Creative Commons Attribution 3.0 License, which permits unrestricted use, distribution, and reproduction in any medium, provided the original work is properly cited. 